\title{
Protée
}

\section{Art réseau et modalités épistolaires}

\section{Joanne Lalonde}

Volume 30, numéro 1, printemps 2002

Les formes culturelles de la communication

URI : https://id.erudit.org/iderudit/006694ar

DOI : https://doi.org/10.7202/006694ar

Aller au sommaire du numéro

Éditeur(s)

Département des arts et lettres - Université du Québec à Chicoutimi

ISSN

0300-3523 (imprimé)

1708-2307 (numérique)

Découvrir la revue

Citer cet article

Lalonde, J. (2002). Art réseau et modalités épistolaires. Protée, 30(1), 7-13. https://doi.org/10.7202/006694ar

\section{Résumé de l'article}

Ce texte propose une réflexion sur les rapports entre les pratiques d'art réseau et les modalités de la tradition épistolaire. À travers l'examen de trois grandes tendances de l'œuvre en réseau, la pratique du mail art, la lettre vidéo et l'œuvre hypermédiatique, nous tenterons de démontrer comment ces œuvres composites (cohabitation de l'image, du texte et du son) renouvellent la tradition épistolaire, faisant passer l'épistolaire d'une tradition littéraire à des formes plus succinctes et interactives. L'essentiel de l'œuvre en réseau vise alors à construire un lien esthétique avec le spectateur sous le modèle de l'interaction dialogique ou polyphonique, directe et fictive. Ainsi, le recours aux modalités de l'épistolaire représente une des stratégies importantes de l'interactivité fictive dans l'art réseau. 


\section{ART RÉSEAU ET MODALITÉS ÉPISTOLAIRES}

JOANNE LALONDE

Ce texte propose une réflexion sur les rapports entre les pratiques d'art réseau ${ }^{1}$

et les modalités de la tradition épistolaire. À travers l'examen de trois grandes tendances de l'œuvre en réseau, la pratique du mail art, la lettre vidéo et l'œuvre hypermédiatique ${ }^{2}$, nous constaterons comment ces œuvres composites (cohabitation de l'image, du texte et du son) renouvellent la tradition épistolaire, faisant passer à première vue l'épistolaire d'une tradition littéraire à des formes plus succinctes, dynamiques et interactives.

En privilégiant la circulation d'un propos visuel, verbal, sonore, l'œuvre réseau s'inscrit dans la tradition de l'epistellein. La notion même d'écriture s'y trouve ainsi élargie, intégrant des stratégies narratives empruntées aux modes d'expression visuel et oral. Cette pratique se trouve alors doublement liée aux moyens de communication: par les outils plus ou moins technologiques qui véhiculent le propos, mais également par les idéologies de production et de diffusion qui en conditionnent les discours.

Il semble important de rappeler tout d'abord que l'histoire de l'art réseau ne se réduit pas à celles des nouvelles technologies des télécommunications, tout particulièrement à celle de l'art hypermédiatique (diffusé sur le web). En effet, la pratique du mail art dans les années 1960, par exemple le projet de On Kawara, I Got $U_{p}(1968-76)^{3}$, représente l'une des activités originaires de celui-ci. Cette précision établie, il est vrai qu'aujourd'hui Internet devient le nouvel emblème de l'art médiatique, le courrier électronique faisant, à première vue, passer l'épistolaire d'une tradition littéraire introspective à des formes plus succinctes qui sont empruntées à la rhétorique de la carte postale ou à celle de la conversation verbale dont elle reproduit l'impression de direct.

Le courrier électronique, comme forme d'expression épistolaire récente, a été associé dès ses débuts à la correspondance d'affaires, mais il est très rapidement devenu populaire et intégré dans les échanges interpersonnels quotidiens.

Différentes études ont permis de constater qu'à travers son exercice, la pratique de l'écriture se trouvait radicalement renouvelée ${ }^{4}$ : coexistence d'éléments linguistiques de langues différentes, utilisation très fréquente d'abréviations parfois oralisantes, de l'ellipse et divers moyens d'expressions graphiques (répétitions de lettres, points 
de suspension ou d'exclamation, caractères différents au sein du même message et autres symboles préprogrammés par les logiciels). Cette vision demeure fragmentaire. En effet, le courrier électronique, comme Internet à une échelle de diffusion générale, peut tout autant véhiculer des formes poétiques et artistiques. Cet outil de diffusion n'est plus uniquement considéré du point de vue de l'efficacité du modèle des télécommunications ${ }^{5}$ et, comme la poste et la vidéo avant lui, il intéresse de nouvelles générations de créateurs.

\section{ESTHÉTIQUE DU LIEN}

L'essentiel de l'œuvre en réseau sera alors de construire un lien esthétique avec le spectateur sous le modèle de l'interactivité directe ou fictive. En effet, les nouvelles images composites propres à cette esthétique demandent parfois une participation accrue du spectateur, qui deviendra un interacteur dans la mesure où il contribuera à l'élaboration du propos de l'œuvre, par exemple lorsque les opérations qu'il déclenche s'intègrent au fur et à mesure dans le processus de l'objet. Cette interaction peut se dérouler sous le modèle du dialogue ou du polyphone, ces dialogue et polyphone pouvant être réels ou fictifs. En ce sens, le recours aux modalités de l'épistolaire représente une des stratégies importantes de l'interactivité fictive dans l'art réseau.

Les trois tendances fondatrices de l'œuvre en réseau - la pratique du mail art, la lettre vidéo, l'œuvre hypermédiatique - seront abordées ici à travers des exemples qui réactualisent la tradition épistolaire. Au sein de cette tradition, les modalités principalement retenues concernent l'importance des formules de l'incipit, séquences d'ouverture du texte qui établissent d'entrée de jeu le pacte épistolaire ${ }^{6}$, et de l'excipit, mettant fin à l'interaction. À ceci s'ajoutent les stratégies du récit à une ou plusieurs voix ainsi que la récurrence des formes réflexives verbales ( «je t'écris cette lettre») ou visuelles (image de l'acte d'écrire et écriture figurativisée), visant à confirmer le statut de scripteur et agissant comme marqueurs de l'épistolaire.
Un des premiers éléments qui distinguent la lettre de la conversation est son caractère prémédité, construit ${ }^{7}$. Les formules de l'incipit y sont souvent fortement typées, on insiste sur les marqueurs déictiques et l'identité du destinataire, suivis généralement de plusieurs éléments coutumiers servant à confirmer le fait d'écriture.

L'énoncé initial est donc mise en scène de l'écriture de la lettre, matérialisation de la relation entre les correspondants et image de la continuité qu' on veut attribuerà cette relation. ${ }^{8}$

Absence et distance sont deux autres éléments propres à la pratique épistolaire 9 . Dans le cas du courrier électronique, la distance coexiste avec l'idée de proximité, dans la mesure où l'échange a un caractère immédiat. L'espace physique de séparation semble éclater au profit de l'effet de direct ${ }^{10}$. Certains marqueurs déictiques ${ }^{11}$ (formules récurrentes de l'incipit) si importants dans la tradition épistolaire seront conservés dans plusieurs œuvres en réseau comme séquence phatique ou transactionnelle. Souvent ces marqueurs s'y retrouvent automatisés, préprogrammés dans le paratexte par les logiciels qui permettent d'ajouter, à travers la rubrique sujet, des informations résumées du contenu de la lettre. Efficace et rapide, le courrier électronique est trop souvent vu comme lieu de l'échange banalisé, de format neutre et davantage lié à l'utilitaire, qui ne conserve de la tradition épistolaire que le matériau, l'écriture souvent télégraphique, alors qu'il peut tout autant être le véhicule d'une proposition plus intime, plus engagée.

\section{ÉCRIRE À UNE OU PLUSIEURS VOIX}

Une fois le pacte épistolaire reconnu, le locuteur peut recourir à différentes modalités d'énonciation. Dans la tradition du roman épistolaire - le roman épistolaire est une forme entre réel et imaginaire où les lettres fictives sont inspirées de l'écriture des lettres réelles, forme fictive de l'épistolaire très voisine des pratiques artistiques en réseau dans la mesure où ces dernières demeurent dans un registre symbolique et non liées à l'utilitaire -, il y a trois grands types de structures qui conditionnent trois grands types 
d'écriture: monodie, lettre en duo et polyphonie. Ces types fréquents dans le discours critique ont notamment été résumés par Marie-Claire Grassi ${ }^{12}$. Rappelons bien sûr que dans les cas présentés ici, la notion d'écriture implique également le son et l'image; il s'agit alors d'une écriture composite et non d'une production strictement verbale.

\section{PREMIER TYPE: LA MONODIE}

Écho d'une seule voix, en une ou plusieurs lettres, l'exemple le plus connu est celui des Lettres portugaises, soliloque plaintif parce que l'amant ne répond pas, où l'écriture se trouve concentrée sur le moi, l'instance énonciative étant au je.

Je vois bien que je vous aime comme une folle; cependantjene me plains point de toute la violence des mouvements de mon cour, je m'accoutumeà ses persécutions, et je ne pourrais vivre sans un plaisir que je découvre, et dont je jouis en vous aimant au milieu de mille douleurs. ${ }^{13}$

I Got Up de Kawara reprend cette stratégie de l'envoi univoque et de la répétition, de même que l'utilisation de la première personne du singulier, opposant toutefois l'aspect laconique et l'écriture minimaliste et publique de la carte postale à la tradition de l'écriture diariste de l'introspection littéraire. Le texte témoigne surtout ici de son caractère fragmentaire ${ }^{14}$. De plus, le dilemme véridicité ou fictionnalité, souvent soulevé dans les études sur l'épistolaire, s'y trouve résolu par la dimension symbolique de l'œuvre d'art. L'effet réaliste l'emporte, la répétition quotidienne du geste, de même que l'aspect référentiel et descriptif du message accentuent la vraisemblance du propos.

Un autre exemple de monodie est la lettre vidéo de Sébastien Pesot, Placebo (1997) ${ }^{15}$ : un jeune homme décrit à une amie son environnement urbain surréaliste. Nous sommes toutefois ici face à un univers fantastique ouvertement revendiqué, la plasticité des images très stylisées leur enlevant tout effet de transparence. Le discours crédible du narrateur contraste fortement avec l'étrangeté du contexte créé par les images; seule la formule épistolaire demeure vraisemblable. Pesot a également recours aux stratégies conventionnelles de la monodie: le texte témoigne de la vision du narrateurénonciateur, l'adresse au «tu» représentant la seule intégration du personnage destinataire de la lettre, l'autre étant le prétexte à ce témoignage autobiographique. Encore une fois l'incipit est ici déterminant. L'énoncé d'ouverture "Ma chère Annie, je m'ennuie beaucoup de toi», récitée en voix off par le narrateur, suffit à installer la forme épistolaire. Même constat pour l'excipit «J'ai hâte de te revoir, viens me visiter si tu peux, je t'embrasse, je pense à toi»; ces formules insistent sur la distance entre les personnages, justifiant ainsi le recours à la lettre.

\section{DEUXIÈME TYPE : LES LETTRES EN DUO}

Lettres de souvenance (1996) ${ }^{16}$ de Chantal DuPont et d'Elisabeth Wörle, sous le modèle par exemple des Lettres d'Abélard et d'Héloïse, mettent en scène deux interlocutrices le temps d'un été; des histoires s'entrecroisent en une correspondance fermée dans une tradition narrative qui fonctionne par alternance des récits, les épistolières se répondent d'une lettre à l'autre.

Le texte visuel et verbal cohabite avec une série d'images et d'atmosphères sonores témoignant, d'une part, de l'histoire familiale des deux personnages et, d'autre part, de leurs occupations quotidiennes et domestiques. Les marqueurs déictiques propres à la lettre y sont très fortement exploités, marqueurs déictiques qui sont également récités, ponctuant l'ouverture des séquences en voix off; ainsi: Montréal le 16 juillet 1995, Ma chère Elizabeth; Barcelone, le 2 août 1995, Ma chère Chantal. De plus, les personnages confirment fréquemment leur statut de scripteur, soit par le mode verbal: "Je t'écris avec un stylo camera, audelà de la page blanche, le temps d'une lettre», insiste Chantal DuPont, soit par l'image, stratégies d'énonciation réflexives qui agissent, ainsi que je l'ai mentionné plus haut, comme marqueur verbal et iconique de l'épistolaire.

Measures of Distance (Mona Hatoum, 1988) ${ }^{17}$ propose, sur le même modèle, une correspondance 
entre une mère et sa fille. La lettre en duo donne une écriture très contrastée et permet de présenter différents portraits, l'opposition des personnages et des visions du monde. C'est la stratégie qu'exploite à plusieurs niveaux Mona Hatoum: confrontation des voix, entre mère et fille; confrontation des cultures orientale-occidentale; confrontation des langues (l'artiste traduit en anglais les lettres de sa mère, dont on entend pourtant en fond sonore la langue originale), mais confrontation aussi au niveau de l'image; superposition de photo et de texte, ce dernier insistant encore une fois sur les marqueurs iconiques de l'épistolaire (l'écriture y est «figurativisée» 18 , transformée en figure-signe: on voit au premier plan la lettre écrite, on voit à travers elle, premier écran de l'image vidéo) et sur les marqueurs verbaux (on entend les formules récitées de l'incipit dans ses versions superposées, qui reprennent l'idée des superpositions d'images). La lettre en duo exploite ici l'idée de confrontation/conciliation entre les personnages.

\section{ÉCRITURE ET ORALITÉ}

Dans les exemples donnés jusqu'à présent, le spectateur demeure exclu de l'échange épistolaire auquel il assiste a posteriori, bien qu'il ait souvent l'impression d'y participer. L'échange est passé, on lui offre le résultat. L'œuvre est une œuvre témoin d'un échange fictionnalisé.

Il est intéressant de constater que dans ces lettres vidéo, les artistes détournent partiellement la tradition d'oralité, si importante dans l'histoire de l'art vidéo, pour orienter la trame narrative vers l'écriture; insistance sur les marqueurs de l'épistolaire plutôt que sur des marqueurs de l'oralité, dont le mode confessionnel serait l'exemple le plus courant et pour lequel on retrouve une énonciation à la première personne du singulier, un sujet intradiégétique, un personnage en gros plan, ainsi que l'adresse directe à la caméra. De plus, l'ambiguité entre réalité et fiction propre au roman épistolaire ${ }^{19}$ s'y trouve également maintenue, ambiguïté alimentée par la tradition vidéo au sein de laquelle l'utilisation de matériel autobiographique ${ }^{20}$, la possibilité de diffusion en direct et l'important aspect documentaire ont favorisé le glissement entre des genres jusqu'alors considérés comme relativement distincts.

\section{TROISIÈME TYPE: LA POLYPHONIE}

Marie-Claire Grassi le décrit comme roman à plusieurs voix ${ }^{21}$ où la pluralité des instances énonciatives permet de moduler les divers points de vue. L'écriture s'y retrouvera plus fragmentée, éclatée, hétérogène.

Les invitations de différentes natures, lancées par l'artiste transdisciplinaire Marie-Suzanne Désilets dans ses œuvres Rapport d'enquête et 100 Idées en l'air ${ }^{22}$, exploitent l'ouverture polyphonique. Dans Rapport d'enquête, une lettre-invitation est lancée sous plusieurs formes: affichage photo, message téléphonique ou courrier électronique, circulation de petits objets à des correspondants potentiels ${ }^{23}$. Les réponses des participants, qui deviennent des correspondants, s'intègrent dans le déroulement des séquences suivantes afin de créer un microcosme relationnel qui reprend la diversité des points de vue décrite par M.-C. Grassi.

Cette proposition vise l'intégration d'un spectateur-interacteur, interacteur parce qu'il aurait une attitude plus active, mais surtout parce que l'œuvre permet ce dispositif en ne se proposant plus comme un produit fini, un objet autonome, mais comme un objet qui aurait plutôt l'aspect dynamique propre à l'échange dans le processus de communication.

Le polyphonique, chez Marie-Suzanne Désilets, implique l'idée d'une collectivité. Collectivité non seulement représentée dans les modèles d'écriture à plusieurs voix, mais une collectivité active dans l'expérience de l'œuvre.

Dans 100 Idées en l'air, une centaine de ballons soufflés à l'hélium sont lancés un par un à partir d'un toit du centre-ville de Montréal. Happening épistolaire, à chacun des ballons est attaché un message sous forme de coupon-réponse ${ }^{24}$. Dans un post-scriptum, l'artiste demande aux récepteurs du ballon de 
retourner le coupon-réponse en s'identifiant et en identifiant le lieu de la réception du message.

\section{L'ÉPISTOLAIRE, PROCÉDÉ DE L'INTERACTIVITÉ}

Cet exemple témoigne du fait que la recherche de l'interactivité n'est pas exclusive à l'œuvre technologique. Tout œuvre d'art est interactive dans la mesure où elle implique la participation du spectateur, parce que le sujet est toujours partie constitutive du monde qu'il se représente ${ }^{25}$. Il faut en revanche reconnaître que la technologie a très souvent été le véhicule de cette interactivité (interface, image vidéo du spectateur intégrée dans l'œuvre, dispositif technique que celui-ci doit actionner, etc.). Très souvent nommés, les procédés de l'interactivité demeurent toutefois très peu contextualisés. Il faudrait maintenant mieux définir ces procédés de l'interactivité dont l'épistolaire devient, comme mentionné plus haut, un des modèles dominants. C'est en ce sens que le moyen de communication devient idéologique ${ }^{26}$, lorsqu'il travaille à occuper réellement ou symboliquement l'espace de la distance propre aux processus de communication ou encore à la faire éclater.

L'œuvre réseau présente un certain nombre de paramètres au spectateur et se révèle à travers une série d'opérations, déclenchées par celui-ci, constituant le parcours (parmi plusieurs possibles) de l'œuvre. Si la mobilité et la variabilité du parcours ne sont pas des propositions exclusives encore une fois à l'œuvre d'art en réseau, ce libre parcours a toutefois ici une forte portée constitutive dans la mesure où il génère l'aspect dynamique du territoire de l'œuvre qui n'est plus fixe. Ainsi l'œuvre, ne pouvant plus faire l'objet d'une première saisie globale, occupe un territoire temporaire sans cesse reconstruit par le parcours du spectateur. Il y a donc transformation du comportement attendu de la part de ce dernier.

L'œuvre hypermédiatique exploite beaucoup ces jeux d'intégration du spectateur. Dans La Bibliothèque des rêves (1998) de Jean-Jacques Rullier, le modèle de la polyphonie est central. L'artiste français demande aux cyberspectateurs de lui envoyer des témoignages, récits de leurs rêves, afin de les inclure dans le développement rhizomatique de son œuvre, qui se présente comme un processus et non comme un objet fini, condition essentielle du polyphonique.

Dans ses deux productions, Figures (1998) et Chagrins (1997) 27, la photographe Élène Tremblay utilise le langage verbal comme moteur au parcours de l'œuvre et l'adresse directe au spectateur. Des phrases comme "Je sais que vous êtes là», "Vous êtes venus me visiter " (Figures) installent d'emblée l'image du dialogue et de l'interaction. Ces énoncés réflexifs demandent au spectateur de confirmer sa présence en cliquant sur le colophon ${ }^{28}$ caché dans la phrase. De plus, le fait que ce parcours ne puisse être généré que par l'activité du spectateur renforce cette idée d'échange direct, d'interactivité effective ou même celle de la conversation médiatique, notamment par l'importance du geste très senti; les images et les textes se dérobent et forcent le spectateur à les chasser, donnant une dimension ludique à l'expérience.

Également dans Figures, plusieurs énoncés apparaissent sous la forme de l'illustration pragmatique, le comment c'est montré, illustrant le contenu propositionnel. Par exemple, la phrase «Je ne fais que passer» défile rapidement, elle est difficile d'atteinte, ou encore le mot rien s'efface progressivement. Même stratégie au niveau des images, les photos présentées agissent en redondance pragmatique du texte. La vigilance du spectateur est sans cesse requise pour progresser dans l'œuvre, d'où l'idée du jeu de chasse évoqué plus haut.

Loin de la complexité de certains jeux vidéo, ces œuvres de Tremblay demeurent dans une logique de la linéarité (diffusion d'informations sur une trajectoire continue et homogène). L'interactivité semble faible à un premier niveau, davantage fictive qu'effective. En revanche, les formes de l'interactivité fictive s'y trouveront confirmées par l'aspect performatif et réflexif de chaque séquence ainsi que par le recours aux modalités de l'épistolaire, ici par le modèle monodique qui implique une réponse, indispensable, nous le savons, au parcours de l'œuvre. 


\section{LA LETTRE COMME OBJET FÉTICHE}

Toutes ces propositions s'inspirent davantage du modèle de la correspondance que de celui de la conversation. Rappelons les éléments de la forme épistolaire fortement exploités par les œuvres en réseau: incipit et excipit typés, énonciation monologique, dialogique ou polyphonique et dimensions réflexives locutionnaire et illocutionnaire ${ }^{29}$. La lettre se distingue également du dialogue dans la mesure où le discours se trouve dédoublé en moment d'écriture et moment de lecture qui appartiennent à des lieux et à des temps différents ${ }^{30}$. Ce qui caractérise parfaitement l'œuvre hypermédiatique, ces moments de lecture étant exponentiellement multipliés. Demeure toutefois cette intention de l'échange propre au dialogue que l'acte de réception de l'œuvre va rendre effectif.

L'œuvre hypermédiatique contourne la dimension privée de la lettre. Elle s'adresse à de multiples allocutaires, la bouteille lancée sur le réseau des réseaux, qui ne sont pas préidentifiés; elle fonctionne comme une lettre ouverte qui jouera davantage sur une finalité externe (la circulation d'un propos artistique, poétique) que sur des enjeux relationnels précis. Tout est public ici, l'énonciateur, l'énonciataire représentent des instances sociales et des locuteurs interchangeables. La mise en scène du moi singulier disparaît au profit de la mise en scène de la relation qui engage les correspondants ${ }^{31}$, une relation témoignant d'interactions à chaque fois uniques.

Mais la lettre, c'est aussi l'absence de l'autre, absence du corps de l'autre, dont elle se présente parfois comme un fétiche ${ }^{32}$, objet transactionnel de substitution. À travers ses formes électroniques, cette immatérialité devient encore plus criante puisque l'objet même est un fantôme et que seule demeurera l'expérience du lien. En contrepartie à cette dissipation, l'oralité maintiendra la présence du corps au sein de l'œuvre. Dans l'art vidéo, elle implique plus que le son de la voix qui raconte. On pourrait la comprendre comme fonction de l'oralité dans l'acte de communication, la trace indicielle d'une voix qui évoque toujours un corps, renvoyant ainsi au sujet existentiel. En proposant le corps également comme un lieu d'inscription du texte, la lettre vidéo en multiplie les portées sémiotiques.

Dans les vidéogrammes présentés plus haut, le corps témoigne toujours de la présence du scripteur dans la lettre. Dans Placebo, la présence du narrateur s'inscrit à travers sa récitation. Dans Measures of Distance, corps et lettre ne font qu'un, fétiche de la présence maternelle dont la lecture en voix off offre un redoublement. Dans Lettres de souvenance, c'est par le corps que se livre l'épistolaire, les marqueurs verbaux (par exemple les formules récitées de l'incipit) et les marqueurs factuels (cette insistance sur le geste d'écriture que l'on retrouve à la fois dans l'image et dans le texte) mettent davantage en scène le cérémoniel de l'échange entre les épistolières qu'ils ne livrent la personnalité de chacune. Le corps devient alors bivalent, témoin à la fois de la séparation qui rend la lettre nécessaire et de l'impression de proximité créée par cette dernière.

\section{NOTES}

1. De manière très générale, l'art réseau se définit comme une pratique artistique dont le processus utilise des moyens de communication (poste, télégramme, téléphone, télécopie), et plus récemment les technologies des télécommunications comme celle bien sûr d'Internet.

2. Voir la définition de l'hypermédia dans Dictionnaire des arts médiatiques, L. Poissant (sous la dir. de), Sainte-Foy, P.U.Q., 1997, p. 166-167, qui implique le principe associatif, une mégastructure interreliant plusieurs informations textuelles et graphiques.

3. Il s'agit de l'envoi quotidien du même message donnant l'heure (réelle... fictive?) du lever de l'artiste sur plusieurs centaines de cartes postales expédiées à différentes personnalités de la scène de l'art contemporain. Les différents projets de mail art de Kawara comptent plus de mille correspondants. 
4. Voir notamment Épistolaire numérique (http://izuminka.free.fr/ mailomanie/), site web conçu par N. Ivanova, dans le cadre d'une maîtrise MST Information et Communication, Université de Paris 8. 5. Le courriel considéré comme le télégramme de la troisième modernité

6. L'incipit épistolaire correspond selon L. Jagueneau aux formules adlocutives (adresse, apostrophe, en-tête, etc.) ainsi qu'aux premières phrases de la lettre. Voir L. Jagueneau, «L'incipit épistolaire: formule adlocutive et énoncé initial dans la correspondance de Francis Jammes et André Gide (1893-1938)», dans L. Louvel (sous la dir. de), L'Incipit, Poitiers, Éd. La Licorne, 1997, p. 291-303.

7. Pourtant S. de Beauvoir et J.-P. Sartre diront le contraire lorsqu'ils la comparent à «la littérature culturelle». Dans La Cérémonie des adieux, "la lettre est une transcription de la vie immédiate», "un travail spontané ", une "littérature brute» "sans visée littéraire» et devenue malgré tout littéraire. Cité par G. Idt et J.-F. Louette, "Voilà de la lettre ou non ", dans A. Magnan (sous la dir. de), Expériences limitées de l'épistolaire, Paris, Champion, 1993, p. 288.

8. L. Jagueneau, op. cit., p. 297.

9. On écrit par ce qu'on est séparé. Voir C. Kerbrat-Orecchioni, "L'interaction épistolaire", dans J. Siess (sous la dir. de), La Lettre entre réel et fiction, Paris, Sedes, 1998. Grâce à la lettre, ajoute B. Melançon, «le présent dysphorique de l'absence est remplacé par la présent euphorique de la lettre»(B. Melançon, SevigneInternet: Remarques sur le courrier électronique et la lettre, Montréal, Fides, 1996, p. 21).

10. Ce qui fait dire à C. Kerbrat-Orecchioni que le courrier électronique, bien qu'il emprunte le canal écrit, s'apparente davantage aux modalités de la communication orale.

11. Adresses des destinataire et destinateur, date et heure de l'envoi.

12. M.-C. Grassi, Lire l'épistolaire, Paris, Dunod, 1998.

13. Extrait tiré de la quatrième lettre: Guilleragues (G.J. de Lavergne), Lettres portugaises, Genève, Librairie Droz, 1972, p. 163.

14. La carte postale, écrit J. Lecourt, n'est pas de "l'image avec de l'écrit [...] c'est de l'écrit en moins". Voir "La carte postale», dans Des Mots et des images pour correspondre, Actes du $2^{\mathrm{e}}$ colloque international, «Les correspondances", Nantes, Université de Nantes, 1986, p. 180.

15. Voir également S. Pesot, Le Paradoxe: une vidéo placebo entre la fiction et la réalité, Montréal, Université du Québec à Montréal, 1999. 16. Le vidéogramme fait partie d'une série de trois bandes (Montréal, 1996). La seconde s'intitule De cour et de parole (1999) et une troisième est à venir.

17. M. Hatoum, Measures of Distance, vidéocassette, Vancouver, Western
Front, 1988.

18. J'emprunte cette expression à M. Sicard, "Correspondances motsimages; autour du groupe COBRA", dans Des Mots et des images pour correspondre, op. cit., p. 2748 .

19. C'est d'ailleurs le titre d'un ouvrage collectif sur le sujet, La Lettre entre réel et fiction, $c f$. note 9 .

20. La vidéo est propice à livrer une version intime des événements. 21. C'est le cas de Julie ou La nouvelle Héloïse de Rousseau, 1761, ou encore celui de la préface de La Religieuse de Diderot, tirée de la correspondance littéraire de Grimm, 1770.

22. Présentées à la galerie Dare Dare de Montréal en 1999 et 2000.

23. L'artiste précise qu'elle travaille sous le modèle de la lettre dans une bouteille lancée à la mer.

24. Le texte est le suivant: «sujet léger pour tête légère, poches aux yeux, poches d'air, gonflé à bloc et léger comme l'air, mots dégonflés pour flotter jusqu'à vous, récipiendaire de cette pensée sur l'air comprimé. Je manque d'air. MSD».

25. Entendu ici comme monde phénoménal.

26. Idéologique est considéré ici dans le sens donné par Barthes dans

"Rhétorique de l'image», Communication, n 4, Paris, Seuil, 1964, p. 49

Les signifiants de connotation représentent le niveau rhétorique et les signifiés de connotation le niveau idéologique du message.

27. Figures (http://www.meduse.org/vuphoto/figures/default.html); Chagrins (http://www.studioxx.org/maidincyberspace/tremblay.html). 28. Expression empruntée à Lacan par F. Recanati: «le colophon, dans un vieux texte, c'est cette petite main indicative qu'on imprimait dans la marge, du temps où on avait encore une typographie" ( $\mathrm{La}$ Transparence et l'Énonciation, Paris, Seuil, 1979, p. 142). Le colophon est employé ici pour décrire l'icône de la petite main désignant le parcours possible au sein de l'œuvre hypermédiatique.

29. Considérées du point de vue d'Austin comme deux aspects liés, le contenu de l'énoncé et l'acte ou le fait de dire ce qui est dit. Voir la présentation de F. Recanati, op.cit.

30. F. Voisin-Atalni, "L'Instance de la lettre», dans La Lettre entre réel et fiction, op. cit., p. 100

31. Alors qu'habituellement ces deux mises en scène coexistent dans le message. Voir M.-F. Chanfrault-Duchet, «Lettres d'adolescentes en détresse : énonciation du moi et représentations sociales ", dans M. Bossis (sous la dir. de), La Lettre à la croisée de l'individuel et du social, Paris, Kimé, 1994, p. 186.

32. Pouvoir fétichiste, voire même religieux, on pourrait alors parler de consubstantiation. 